\section{Eventos adversos pós-vacinação contra influenza pandêmica A (H1N1) 2009 em crianças}

\author{
Adverse events following vaccination against \\ pandemic influenza A (H1N1) 2009 in children
}

\section{Abstract}

The aim of this study was to estimate the frequency of adverse events following vaccination against pandemic influenza A (H1N1) 2009 and associated factors in children from six months to two years of age $(n=156)$. Multivariate Cox regression was used to assess the independent associations between covariates and complaints of at least one adverse event. Strength of association was measured by hazard ratios and respective 95\% confidence intervals. Following the first dose, $40.3 \%$ of parents reported one or more adverse events in their children, compared to $35.5 \%$ after the second dose. Systemic adverse events, specifically irritation, diarrhea, and fever, were more frequent than local reactions at the vaccination site. Incidence rates for adverse events in general and systemic reactions following the first dose were higher in children with concomitant illness or allergies $(H R=3.43,95 \% C I: 1.34-8.77$ and $H R=2.76$, 95\%CI: 1.11-6.89). Most events were mild. Cases of high fever, vomiting, and diarrhea prompted parents to seek care for their children at health services.

Influenza A Virus, H1N1 Subtype; Vaccines; Adverse Effects; Child
Gisele Nepomuceno de Andrade 1

Adriano Marçal Pimenta 1

Débora Arreguy Silva 1

Anézia Moreira Faria Madeira 1

\section{Introdução}

A influenza, comumente conhecida como gripe, é uma infecção viral aguda do sistema respiratório, de distribuição global e elevada transmissibilidade. Os vírus influenza tipo A são mais vulneráveis a variações antigênicas, sendo responsáveis pela ocorrência da maioria das epidemias e os únicos com poder de causar a doença no nível de pandemia 1,2. No primeiro semestre de 2009, a Organização Mundial da Saúde (OMS) declarou Emergência em Saúde Pública de Importância Internacional devido à ocorrência de casos humanos de doença respiratória aguda grave causada por um novo subtipo A, o (H1N1), com disseminação entre humanos e em diferentes continentes do mundo 3,4 . Esta situação cumpria o critério para definição de pandemia, sendo a primeira no século XXI. No mundo, mais de 200 países confirmaram casos da influenza pandêmica A (H1N1) 2009, dentre eles o Brasil 5 .

Neste contexto, a vacinação em grande escala foi utilizada como estratégia para controlar a pandemia de influenza A (H1N1) 2009 em vários países, considerando-se que ela poderia diminuir a morbimortalidade e transmissão da doença entre seres humanos 2,6,7. A introdução de uma nova vacina para responder à pandemia tornou-se algo essencial, entretanto, o seu perfil de segurança era menos certo que o de vacinas já existentes contra a influenza ${ }^{8,9}$. Esse fato tornou- 
se uma grande preocupação do público de diferentes países 7,10 .

No Brasil, incluíram-se nos grupos prioritários a serem vacinados, crianças com idade entre seis meses e dois anos pelo fato das taxas de hospitalização, na vigência da pandemia, terem sido altas nesta faixa etária 3 . Ensaios clínicos, com a participação de crianças, realizados para estimar a eficácia e a segurança das vacinas contra a influenza A (H1N1) 2009 e as notificações recebidas de países que realizaram a vacinação antes do Brasil, indicaram que esses imunobiológicos eram tão seguros quanto os da influenza sazonal 11,12,13,14. Entretanto, estudos sobre os eventos adversos pós-vacinação contra a influenza ainda são escassos no Brasil, principalmente, os que incluem crianças, uma vez que estas somente receberem a vacina anual do sistema público de saúde se pertencerem aos grupos de risco para complicações da doença 15,16.

Assim, o presente estudo teve por objetivo estimar a frequência e os fatores associados à ocorrência de eventos adversos pós-vacinação contra a influenza pandêmica A (H1N1) 2009 em crianças com idade entre seis meses e dois anos.

\section{Métodos}

Estudo epidemiológico de delineamento longitudinal, descritivo e exploratório, realizado em um centro de saúde da regional nordeste do $\mathrm{Mu}$ nicípio de Belo Horizonte, Minas Gerais, Brasil, que possuía em sua área de abrangência, aproximadamente, 20 mil habitantes considerados de médio e alto risco quanto ao índice de vulnerabilidade à saúde.

A população do estudo constituiu-se de todas as crianças com idade entre seis meses e dois anos que compareceram ao centro de saúde para a campanha de imunização. No Brasil, a estratégia nacional de vacinação contra a influenza A (H1N1) 2009 foi desenvolvida em cinco etapas com definição de grupos prioritários, sendo que a imunização de crianças na faixa etária do estudo foi realizada no primeiro semestre de 2010, de 22 de março a 2 de abril (la dose) e 26 de abril a 7 de maio (2a dose).

As vacinas utilizadas nos sujeitos do estudo foram adquiridas pelo Ministério da Saúde dos laboratórios Sanofi Pasteur/Butantan e Novartis. Elas eram monovalentes, sem adjuvantes e compostas por vírus inativados propagados em ovos embrionados de galinha, cepa análoga a A/ California/07/2009 (H1N1). Esse imunobiológico foi administrado no músculo vasto lateral da coxa em duas doses correspondente a $0,25 \mathrm{~mL}$ cada, com um mês de intervalo entre elas.
Na época da campanha vacinal, 259 crianças entre seis meses e dois anos de idade residiam na área de abrangência do centro de saúde. Dessas, 193 (74,5\%) procuraram o serviço para receber a vacina contra a influenza A (H1N1) 2009, das quais foram excluídas 37 pelos seguintes motivos: (a) receberam outros imunobiológicos concomitantes às duas doses da vacina $(n=13)$; (b) não foram localizadas para o seguimento $(n=10)$; (c) antes de se vacinar, já apresentavam sintomas clínicos que poderiam interferir nos resultados do trabalho $(n=14)$. Assim, $156(60,2 \%)$ crianças constituíram a população do estudo, das quais 27 receberam outros imunobiológicos junto com a la dose da vacina e 63, junto com a 2a dose. Portanto, a amostra final para seguimento após a 1a dose foi de 129 (49,8\%) crianças e após a 2a dose, de 93 (35,9\%) (Figura 1).

Os dados foram coletados por entrevistadores previamente capacitados em um treinamento que teve duração de oito horas, no qual foram abordadas questões conceituais e práticas sobre a temática do estudo, além do detalhamento de todo o processo de organização e execução da coleta de dados. Esse treinamento foi ministrado pela coordenadora da pesquisa que, também, foi a responsável pela supervisão da coleta de dados que aconteceu em três momentos distintos.

- 1o momento: as crianças que compareceram ao centro de saúde para a imunização com a primeira dose da vacina foram convidadas a participar da pesquisa por meio de seus pais ou responsáveis após serem informados sobre os objetivos do estudo. A elegibilidade de cada criança para participar da pesquisa foi determinada de acordo com os critérios de exclusão já citados. Os pais ou responsáveis autorizaram a participação mediante assinatura do Termo de Consentimento Livre e Esclarecido. Após o consentimento, foram coletados, em formulário próprio, dados sobre as características demográficas da criança, telefone de contato dos pais ou responsáveis e o horário de preferência para a chamada. Em seguida, os pais ou responsáveis foram orientados a monitorar os eventos adversos locais e sistêmicos que viessem a ocorrer após a vacinação, por um período de seis dias, registrando, sobre cada evento adverso, as datas de início e término, e as intervenções realizadas. Por se tratar de uma avaliação mais subjetiva, foi explicado aos pais ou responsáveis como observar a dor ao manipular, o edema, o nódulo e a irritabilidade, eventos adversos esperados após a vacinação contra a influenza A (H1N1) 200911. Em relação à febre, os pais ou responsáveis foram orientados a monitorar a temperatura com o auxílio do termômetro, quando possível, ao menos uma vez ao dia. 
Figura 1

Seleção da população em estudo no geral e após cada dose da vacina contra influenza A (H1N1) 2009. Belo Horizonte, Minas Gerais, Brasil, 2010.

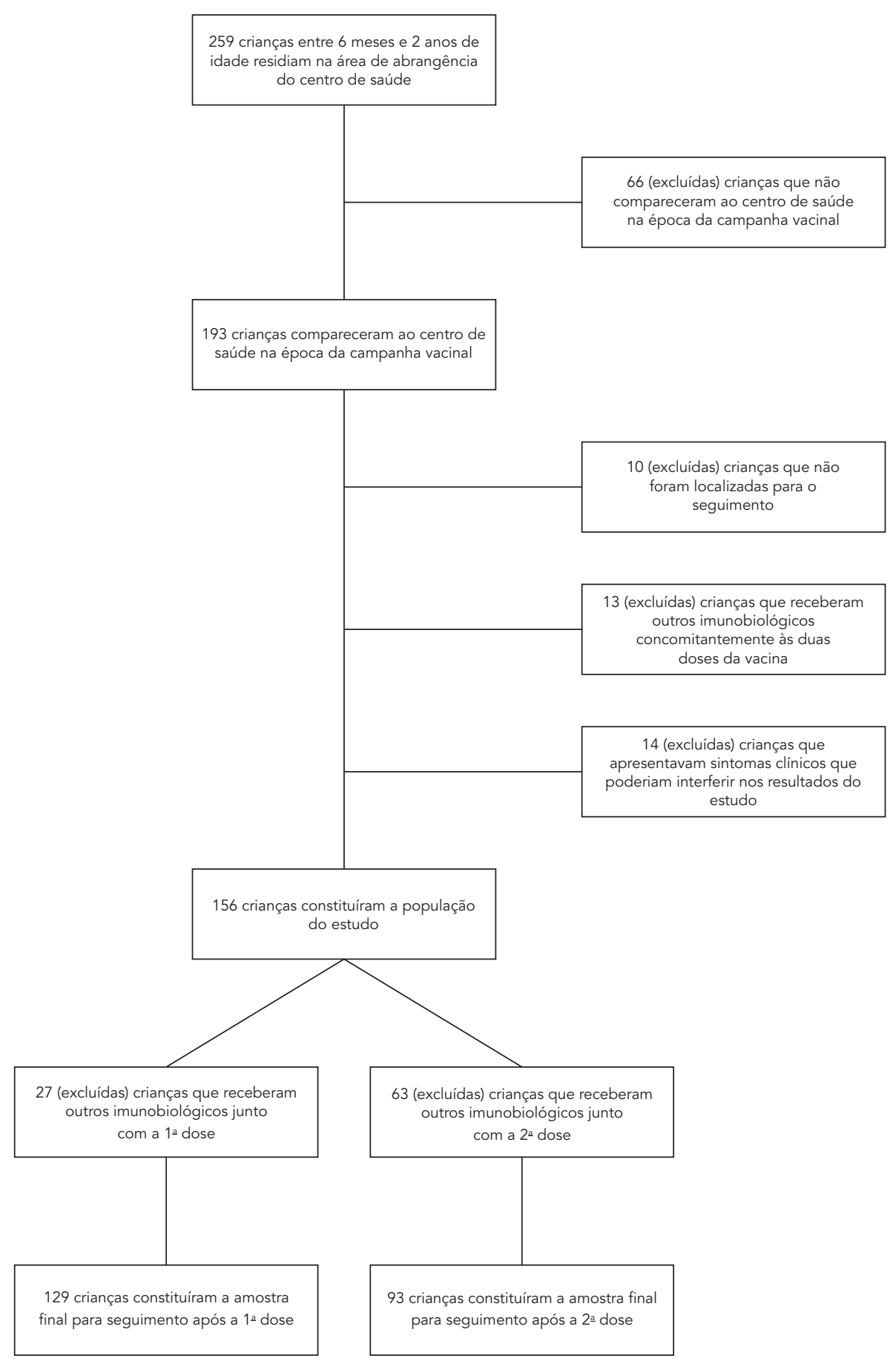


- 2o momento: os pais ou responsáveis pelos sujeitos do estudo foram contatados por telefone para a investigação da ocorrência de eventos adversos seis a nove dias após a vacinação. Os eventos adversos ocorridos foram relatados espontaneamente pelos pais ou responsáveis aos pesquisadores, bem como o tempo de duração e as intervenções realizadas. Os pesquisadores utilizaram formulário próprio com campos abertos e fechados, confeccionado com base nas informações da ficha de notificação padrão do Programa Nacional de Imunização 17, do Protocolo de Vigilância Epidemiológica de Eventos Adversos Pós-Vacinação contra o Vírus da Influenza Pandêmica A (H1N1) 200911 e informações do Manual de Vigilância Epidemiológica de Eventos Adversos Pós-Vacinação ${ }^{18}$, para registrar as informações fornecidas pelos pais ou responsáveis, classificar a intensidade do evento adverso relatado e categorizá-lo como local ou sistêmico.

- 3o momento: a imunização com a segunda dose foi agendada para trinta dias após a administração da primeira. Realizou-se a confirmação do consentimento dado pelos pais ou responsáveis e, o processo de coleta de dados foi o mesmo descrito anteriormente para a primeira dose da vacina.

O conceito de evento adverso adotado no estudo é definido como sendo qualquer ocorrência clínica indesejável em indivíduo que tenha recebido algum imunobiológico 11,18,19.

A intensidade do evento foi classificada da seguinte maneira: (a) eventos graves foram os que resultaram em hospitalização por mais de 24 horas, independente do tipo de evento, disfunção ou incapacidade significativa e risco de morte; (b)eventos moderados foram os que necessitaram de avaliação médica e causaram desconforto na manutenção das atividades diárias da criança; (c) eventos leves foram os que não necessitaram de avaliação médica e não alteraram as atividades habituais da criança. Para a febre a intensidade foi leve $\left(\geq 37,5^{\circ} \mathrm{C}\right.$ a $\left.\leq 38,5^{\circ} \mathrm{C}\right)$, moderada $(\geq$ $\left.38,5^{\circ} \mathrm{C} \mathrm{a} \leq 39,5^{\circ} \mathrm{C}\right)$ e grave $\left(>39,5^{\circ} \mathrm{C}\right) 18$.

A variável dependente do estudo foi a queixa de pelo menos um evento adverso (no geral e categorizado em eventos locais e sistêmicos). Os eventos locais foram definidos como manifestações no local de administração do imunobiológico, enquanto os eventos sistêmicos, como manifestações que afetaram o organismo da criança de forma total, seguindo as orientações do Manual de Vigilância Epidemiológica de Eventos Adversos Pós-Vacinação 18.

As covariáveis foram: sexo (masculino, feminino), idade (6 a 11 meses, 12 a 24 meses), cor da pele (branca, negra/parda) e doença concomi- tante/alergia (sim, não). Essas variáveis foram selecionadas para análise dos fatores associados à ocorrência de eventos adversos após a vacinação por serem possíveis determinantes do desfecho já descritos na literatura por diferentes autores 6,13 e pelo Ministério da Saúde 11 em relação a outros imunobiológicos. A variável doença concomitante/alergia é resultante da seguinte questão do formulário de coleta de dados: a criança possui alguma doença (alérgica ou não) previamente diagnosticada por um médico?

Um banco de dados foi construído com o auxílio do software Epi Info versão 3.5.1 (Centers for Disease Control and Prevention, Atlanta, Estados Unidos) e analisado com o software SPSS versão 15.0 (SPSS Inc., Chicago, Estados Unidos).

A amostra foi caracterizada com a distribuição de frequências absolutas e relativas das variáveis.

A incidência dos eventos adversos foi calculada tendo como denominador todas as crianças vacinadas em cada etapa da campanha. $\mathrm{O}$ numerador foi construído de duas maneiras: (a) para o total de eventos adversos, o número de crianças acometidas pelos desfechos locais e/ou sistêmicos; (b) para os eventos adversos locais e sistêmicos individualmente, o número total de ocorrências de cada um deles.

Diferenças estatísticas entre a ocorrência de eventos adversos após cada dose da vacina, segundo as categorias das covariáveis foram avaliadas com o uso dos testes de qui-quadrado de Pearson ou exato de Fisher.

Hazard ratios (HR) e seus intervalos de 95\% de confiança (IC95\%) foram calculados com a regressão multivariada de Cox, a fim de estimar as associações independentes entre as covariáveis e a queixa de eventos adversos (no global e separadamente como locais ou sistêmicos) após cada uma das doses.

O nível de significância estatística foi fixado em $5 \%(p<0,05)$.

O estudo foi submetido e aprovado pelos Comitês de Ética em Pesquisa da Universidade Federal de Minas Gerais (protocolo: 019/10) e da Secretaria Municipal de Saúde de Belo Horizonte (parecer: 0019.0.410. 203-10A).

\section{Resultados}

A maioria das crianças, no geral, era do sexo masculino (55,1\%), tinha entre 6 e 11 meses de idade $(51,3 \%)$ e a cor de pele negra/parda $(61,5 \%)$. Com exceção da faixa etária, cujas crianças selecionadas após a segunda dose da vacina tinham mais frequentemente idades entre 12 e 24 meses $(55,9 \%)$, as demais características foram simila- 
res em ambos os grupos de participantes incluídos em cada etapa da campanha vacinal.

Todos os sujeitos residiam na área de abrangência do centro de saúde na época do estudo e, seus pais ou responsáveis possuíam algum tipo de linha telefônica fixa e/ou móvel para o contato, e não se recusaram a participar da pesquisa.

Com relação à variável doença concomitante/ alergia, 21,7\% dos sujeitos possuíam alguma doença diagnosticada na época do estudo, sendo que $79,4 \%$ eram doenças do sistema respiratório [sinusite $(11,1 \%)$, bronquite $(22,2 \%)$ e asma $(66,6 \%)]$ e $20,5 \%$ eram alergias dermatológica, alimentar e medicamentosa.

As crianças excluídas da análise, da primeira dose, por terem recebido juntamente outro imunobiológico $(\mathrm{n}=27)$ apresentavam características demográficas semelhantes àquelas que permaneceram no estudo desta etapa. Por outro lado, as crianças excluídas da segunda dose em decorrência do mesmo motivo $(n=63)$ eram proporcionalmente mais jovens (idades entre 6 e 11 meses) do que aquelas que permaneceram no estudo desta etapa $(\mathrm{p}<0,05)$.
Na Tabela 1 são mostrados os tipos de eventos adversos pós-vacinação contra a influenza A (H1N1) 2009 que acometeram as crianças após cada dose, bem como a frequência de cada um. Os tipos de eventos adversos referidos foram os mesmos na primeira e na segunda dose, variando apenas a frequência, que foi maior após a primeira dose, exceto a dor ao manipular.

Após a primeira dose, foi relatado algum tipo de evento adverso por $40,3 \%$ dos participantes e, após a segunda dose, por 35,5\%. Os eventos adversos sistêmicos foram mais frequentes que os locais em ambas as doses, com destaque para a irritabilidade, a diarreia e a febre (Tabela 1).

Ademais, na maioria das vezes, as crianças apresentaram apenas um evento adverso após cada etapa da vacinação, não sendo observada a ocorrência simultânea de mais do que quatro desfechos (Tabela 1).

Nas Tabelas 2 e 3 são apresentadas, para a primeira e segunda dose respectivamente, a distribuição das crianças com evento adverso pósvacinação (no geral e categorizado em eventos locais e eventos sistêmicos) segundo as covari-

Tabela 1

Distribuição da frequência dos eventos adversos pós-vacinação contra influenza A (H1N1) 2009 segundo a dose administrada. Belo Horizonte, Minas Gerais, Brasil, 2010.

\begin{tabular}{|c|c|c|c|c|}
\hline \multirow[t]{2}{*}{ Evento adverso * } & \multicolumn{2}{|c|}{ Primeira dose } & \multicolumn{2}{|c|}{ Segunda dose } \\
\hline & n/Total & $\%$ & n/Total & $\%$ \\
\hline Algum tipo de evento adverso ** & $52 / 129$ & 40,3 & $33 / 93$ & 35,5 \\
\hline $1 \star \star \star$ & $30 / 129$ & 23,3 & $24 / 93$ & 25,8 \\
\hline $2 \star \star \star$ & $15 / 129$ & 11,6 & $7 / 93$ & 7,5 \\
\hline $3 * \star \star$ & $5 / 129$ & 3,9 & $2 / 93$ & 2,2 \\
\hline $4^{\star \star \star}$ & $2 / 129$ & 1,6 & $0 / 93$ & 0,0 \\
\hline Eventos locais ** & $22 / 129$ & 17,1 & $10 / 93$ & 10,8 \\
\hline Dor ao manipular \# & $7 / 129$ & 5,4 & $6 / 93$ & 6,4 \\
\hline Vermelhidão no local \# & $9 / 129$ & 6,9 & $5 / 93$ & 5,3 \\
\hline Edema \# & $7 / 129$ & 5,4 & $2 / 93$ & 2,1 \\
\hline Nódulo \# & $8 / 129$ & 6,2 & $2 / 93$ & 2,1 \\
\hline Eventos sistêmicos ** & $47 / 129$ & 36,5 & $32 / 93$ & 34,4 \\
\hline Febre \# & $24 / 129$ & 18,6 & $15 / 93$ & 16,1 \\
\hline Irritabilidade \# & $32 / 129$ & 24,8 & $14 / 93$ & 15,0 \\
\hline Diarreia \# & $22 / 129$ & 17,0 & $10 / 93$ & 10,7 \\
\hline Perda de apetite \# & $13 / 129$ & 10,0 & $5 / 93$ & 5,3 \\
\hline Náusea/Vômito \# & $11 / 129$ & 8,5 & $2 / 93$ & 2,1 \\
\hline
\end{tabular}

* Mais de um evento adverso foi referido por alguns indivíduos;

** A frequência foi calculada tendo como numerador o número de crianças com o evento adverso;

*** A frequência foi calculada a partir da quantidade de eventos adversos identificados em uma mesma criança. Não foi observada a ocorrência de mais de 4 eventos adversos;

\# A frequência foi calculada tendo como numerador a quantidade de vezes que o evento adverso foi relatado. 
Distribuição da queixa de algum tipo de evento adverso e dos eventos locais e sistêmicos pós-vacinação contra influenza A (H1N1) 2009 na primeira dose, por sexo, faixa etária, cor da pele e doença concomitante/alergia. Belo Horizonte, Minas Gerais, Brasil, 2010.

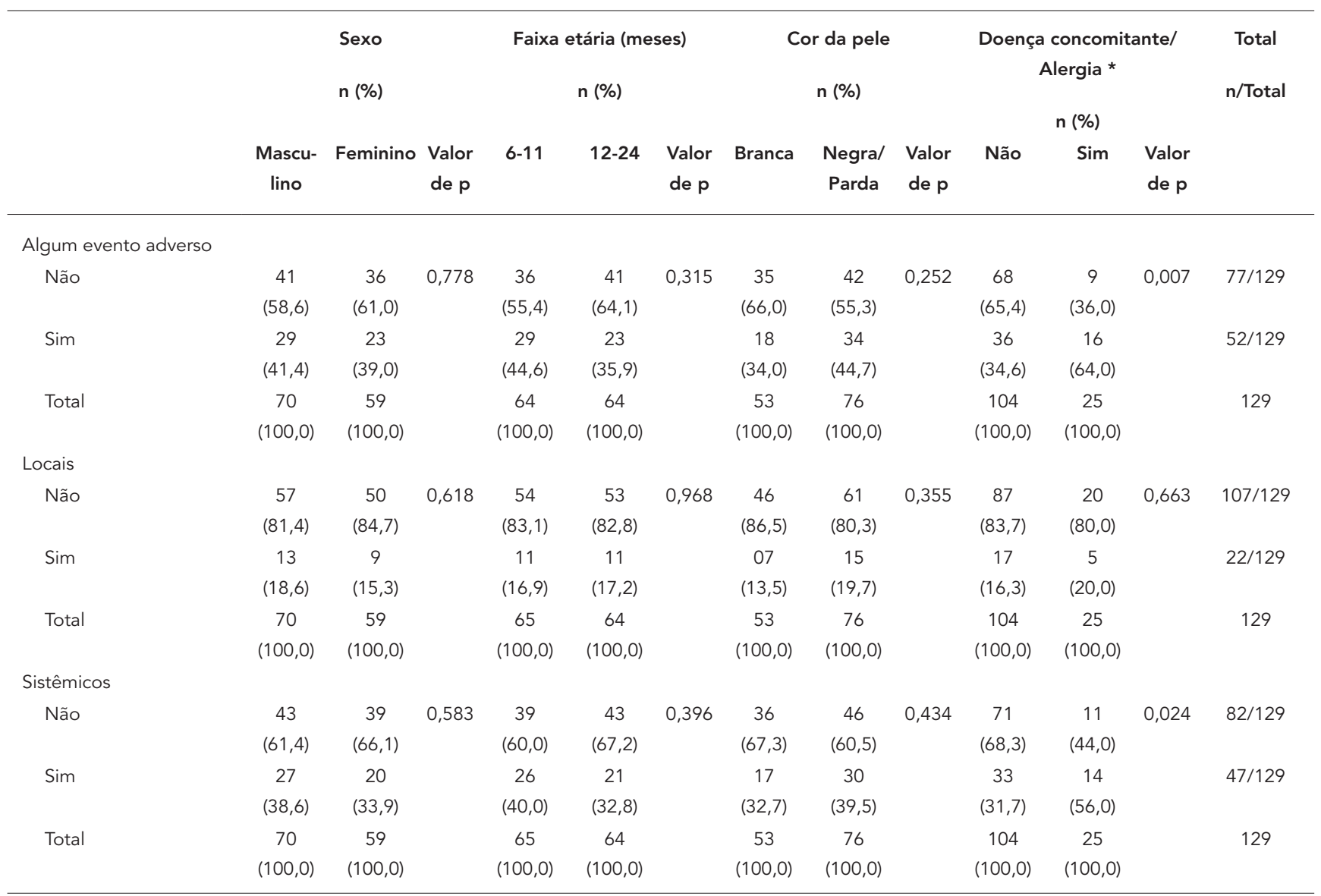

* $80 \%$ doenças do sistema respiratório (sinusite, bronquite e asma), 20\% se distribuem entre alergias dermatológicas, alimentares e medicamentosas.

Nota: valores de $\mathrm{p}$ relativos aos testes de qui-quadrado de Pearson ou exato de Fisher.

áveis. Na primeira dose, as incidências de algum tipo de evento adverso $(\mathrm{p}=0,007)$ e de eventos adversos sistêmicos ( $p=0,024)$ foram maiores em crianças com doença concomitante/alergia (Tabela 2). Na segunda dose, a incidência de eventos sistêmicos foi maior no sexo feminino ( $\mathrm{p}=0,031$ ) (Tabela 3).

Na Tabela 4 são apresentados os modelos de regressão de Cox, construídos para avaliar as associações independentes entre as covariáveis e a incidência de algum tipo de evento adverso (no geral e categorizado em eventos locais e sistêmicos). Após a primeira dose da vacina, a presença de doença concomitante/alergia na criança permaneceu associada à incidência de eventos adversos no geral ( $\mathrm{HR}=3,43$; IC95\%: 1,34-8,77) e sistêmicos (HR = 2,76; IC95\%: 1,11-6,89). No que diz respeito à segunda dose da vacina, o sexo feminino associou-se positivamente à incidência de eventos adversos no geral ( $\mathrm{HR}=2,80$; IC95\%: 1,10-7,16) e sistêmicos (HR = 3,29; IC95\%: 1,25-8,68).

Em relação à intensidade dos eventos adversos pós-vacinação contra a influenza A (H1N1) 2009, houve dois relatos de ocorrências graves na amostra estudada. O primeiro foi de uma criança do sexo feminino, 17 meses de idade, que apresentou episódio de febre maior que $39,5^{\circ} \mathrm{C}$, vômito, diarreia e prostração, sintomas iniciados nas primeiras 24 horas após a imunização com a primeira dose da vacina, com duração de quatro dias. A criança foi hospitalizada, sendo diagnosticado gastroenterite viral. Ela se recuperou completamente em cinco dias. Os pesquisadores não consideraram esse evento relacionado à vacinação. $\mathrm{O}$ segundo foi de uma criança do sexo masculino, vinte meses de idade, que apresentou febre maior que $39,5^{\circ} \mathrm{C}$ (maior pico $39,8^{\circ} \mathrm{C}$ ), 
Distribuição da queixa de algum tipo de evento adverso e dos eventos locais e sistêmicos pós-vacinação contra influenza A (H1N1) 2009 na segunda dose, por sexo, faixa etária, cor da pele e doença concomitante/alergia. Belo Horizonte, Minas Gerais, Brasil, 2010.

\begin{tabular}{|c|c|c|c|c|c|c|c|c|c|c|c|c|c|}
\hline & \multirow{2}{*}{\multicolumn{2}{|c|}{$\begin{array}{l}\text { Sexo } \\
\text { n (\%) }\end{array}$}} & \multicolumn{4}{|c|}{ Faixa etária (meses) } & \multicolumn{3}{|c|}{ Cor da pele } & \multicolumn{3}{|c|}{$\begin{array}{c}\text { Doença concomitante/ } \\
\text { Alergia * }\end{array}$} & \multirow{4}{*}{$\begin{array}{l}\text { Total } \\
\text { n/Total }\end{array}$} \\
\hline & & & & & n (\%) & & & n (\%) & & & & & \\
\hline & \multirow[b]{2}{*}{$\begin{array}{l}\text { Mascu- } \\
\text { lino }\end{array}$} & \multirow[b]{2}{*}{ Feminino } & \multirow[b]{2}{*}{$\begin{array}{l}\text { Valor } \\
\text { de } p\end{array}$} & \multirow[b]{2}{*}{$6-11$} & \multirow[b]{2}{*}{$12-24$} & \multirow[b]{2}{*}{$\begin{array}{l}\text { Valor } \\
\text { de } p\end{array}$} & \multirow[b]{2}{*}{ Branca } & \multirow[b]{2}{*}{$\begin{array}{l}\text { Negra/ } \\
\text { Parda }\end{array}$} & \multirow[b]{2}{*}{$\begin{array}{l}\text { Valor } \\
\text { de } p\end{array}$} & \multirow[b]{2}{*}{ Não } & \multicolumn{2}{|l|}{ n (\%) } & \\
\hline & & & & & & & & & & & Sim & $\begin{array}{l}\text { Valor } \\
\text { de } p\end{array}$ & \\
\hline \multicolumn{14}{|c|}{ Algum evento adverso } \\
\hline \multirow[t]{2}{*}{ Não } & 38 & 22 & 0,052 & 24 & 36 & 0,285 & 19 & 41 & 0,182 & 51 & 9 & 0,152 & $60 / 93$ \\
\hline & $(73,1)$ & $(53,7)$ & & $(58,5)$ & $(69,2)$ & & $(55,9)$ & $(69,5)$ & & $(68,0)$ & $(50,0)$ & & \\
\hline \multirow[t]{2}{*}{ Sim } & 14 & 19 & & 17 & 16 & & 15 & 18 & & 24 & 9 & & $33 / 93$ \\
\hline & $(26,9)$ & $(46,3)$ & & $(41,5)$ & $(30,8)$ & & $(44,1)$ & $(30,5)$ & & $(32,0)$ & $(50,0)$ & & \\
\hline \multirow[t]{2}{*}{ Total } & 52 & 41 & & 41 & 52 & & 34 & 59 & & 75 & 18 & & 93 \\
\hline & $(100,0)$ & $(100,0)$ & & $(100,0)$ & $(100,0)$ & & $(100,0)$ & $(100,0)$ & & $(100,0)$ & $(100,0)$ & & \\
\hline \multicolumn{14}{|l|}{ Locais } \\
\hline \multirow[t]{2}{*}{ Não } & 48 & 36 & 0,466 & 36 & 48 & 0,466 & 29 & 55 & 0,213 & 68 & 16 & 0,819 & $84 / 93$ \\
\hline & $(92,3)$ & $(87,8)$ & & $(87,8)$ & $(92,3)$ & & $(85,3)$ & $(92,3)$ & & $(90,7)$ & $(88,9)$ & & \\
\hline \multirow[t]{2}{*}{ Sim } & 4 & 5 & & 5 & 4 & & 5 & 4 & & 7 & 2 & & $9 / 93$ \\
\hline & $(7,7)$ & $(12,2)$ & & $(12,2)$ & $(7,7)$ & & $(14,7)$ & $(6,8)$ & & $(9,3)$ & $(11,1)$ & & \\
\hline \multirow[t]{2}{*}{ Total } & 52 & 41 & & 41 & 52 & & 34 & 59 & & 75 & 18 & & 93 \\
\hline & $(100,0)$ & $(100,0)$ & & $(100,0)$ & $(100,0)$ & & $(100,0)$ & $(100,0)$ & & $(100,0)$ & $(100,0)$ & & \\
\hline \multicolumn{14}{|c|}{ Sistêmicos } \\
\hline \multirow[t]{2}{*}{ Não } & 39 & 22 & 0,031 & 24 & 37 & 0,204 & 19 & 42 & 0,135 & 52 & 9 & 0,121 & $61 / 93$ \\
\hline & $(75,0)$ & $(53,7)$ & & $(58,5)$ & $(71,2)$ & & $(55,9)$ & $(71,2)$ & & (69.3) & $(50,0)$ & & \\
\hline \multirow[t]{2}{*}{ Sim } & 13 & 19 & & 17 & 15 & & 15 & 17 & & 23 & 9 & & $32 / 93$ \\
\hline & $(25,0)$ & $(46,3)$ & & $(41,5)$ & $(28,8)$ & & $(44,1)$ & $(28,8)$ & & $(30,7)$ & $(50,0)$ & & \\
\hline \multirow[t]{2}{*}{ Total } & 52 & 41 & & 41 & 52 & & 34 & 59 & & 75 & 18 & & 93 \\
\hline & $(100,0)$ & $(100,0)$ & & $(100,0)$ & $(100,0)$ & & $(100,0)$ & $(100,0)$ & & $(100,0)$ & $(100,0)$ & & \\
\hline
\end{tabular}

* $80 \%$ doenças do sistema respiratório (sinusite, bronquite e asma), 20\% se distribuem entre alergias dermatológicas, alimentares e medicamentosas. Nota: valores de p relativos aos testes de qui-quadrado de Pearson ou exato de Fisher.

vômito e diarreia, sintomas iniciados oito horas após a imunização com a primeira dose da vacina. A criança foi atendida por pediatra particular que não identificou causa específica e a medicou com antitérmico, antiemético e soro de reidratação oral. Os sintomas cessaram completamente no terceiro dia após a vacinação.

A maioria dos eventos adversos relatados foi de intensidade leve (89,7\%), ou seja, não prejudicaram as atividades diárias habituais das crianças, não sendo necessário tratamento de saúde. Além disso, esses eventos surgiram dentro de três dias após a vacinação e 80\% deles desapareceram no prazo de dois dias. Foram classificados como moderados $9,8 \%$ dos eventos adversos relatados. A procura por serviços de saúde em decorrência dos eventos foi de $5,1 \%(8 / 156)$ e estavam relacionados à febre entre $39,0^{\circ} \mathrm{C}$ a $39,8^{\circ} \mathrm{C}$, ao vômito e à diarreia.

\section{Discussão}

A incidência de eventos adversos no geral após a primeira dose foi maior do que após a segunda dose da vacina $(40,3 \% v s$. $35,5 \%)$, sendo os sistêmicos mais comuns do que os locais, independentemente da etapa da campanha vacinal. Por outro lado, a maioria dos eventos foi classificada com intensidade leve. Ademais, os eventos adversos no geral e os sistêmicos permaneceram independentemente associados à presença de doença concomitante/alergia após a primeira dose e ao sexo feminino após a segunda dose.

Os eventos adversos pós-vacinação contra a influenza pandêmica A (H1N1) 2009 observados nos participantes do estudo, a frequência e intensidade com que ocorreram foram similares aos observados em ensaios clínicos 13,14,20, meta-análise 21 e estudos observacionais 22,23 que 
Modelos de regressão de Cox para o ajuste multivariado das associações entre as covariáveis e a queixa de algum evento adverso, eventos locais e sistêmicos pós-vacinação contra influenza A (H1N1) 2009, segundo a dose administrada. Belo Horizonte, Minas Gerais, Brasil, 2010.

\begin{tabular}{|c|c|c|c|c|c|c|}
\hline \multirow[t]{2}{*}{ Variáveis } & \multicolumn{3}{|c|}{ Primeira dose } & \multicolumn{3}{|c|}{ Segunda dose } \\
\hline & $\mathrm{HR}$ & IC95\% & Valor de $p$ & HR & IC95\% & Valor de $p$ \\
\hline \multicolumn{7}{|c|}{ Algum evento adverso } \\
\hline \multicolumn{7}{|l|}{ Sexo } \\
\hline Masculino & 1,00 & & & 1,00 & & \\
\hline Feminino & 0,94 & $0,45-1,97$ & 0,877 & 2,80 & $1,10-7,16$ & 0,031 \\
\hline \multicolumn{7}{|l|}{ Idade (meses) } \\
\hline $6-11$ & 1,00 & & & 1,00 & & \\
\hline $12-24$ & 0,56 & $0,26-1,19$ & 0,128 & 0,53 & $0,21-1,34$ & 0,178 \\
\hline \multicolumn{7}{|l|}{ Cor da pele } \\
\hline Branca & 1,00 & & & 1,00 & & \\
\hline Negra/Parda & 1,51 & $0,70-3,26$ & 0,290 & 0,59 & $0,24-1,47$ & 0,254 \\
\hline \multicolumn{7}{|c|}{ Doença concomitante/Alergia * } \\
\hline Não & 1,00 & & & 1,00 & & \\
\hline $\operatorname{Sim}$ & 3,43 & $1,34-8,77$ & 0,010 & 2,40 & $0,80-7,20$ & 0,117 \\
\hline \multicolumn{7}{|l|}{ Eventos locais } \\
\hline \multicolumn{7}{|l|}{ Sexo } \\
\hline Masculino & 1,00 & & & 1,00 & & \\
\hline Feminino & 0,79 & $0,31-2,01$ & 0,620 & 1,82 & $0,43-7,78$ & 0,419 \\
\hline \multicolumn{7}{|l|}{ Idade (meses) } \\
\hline $6-11$ & 1,00 & & & 1,00 & & \\
\hline $12-24$ & 0,93 & $0,36-2,36$ & 0,872 & 0,55 & $0,13-2,36$ & 0,425 \\
\hline \multicolumn{7}{|l|}{ Cor da pele } \\
\hline Branca & 1,00 & & & 1,00 & & \\
\hline Negra/Parda & 1,57 & $0,58-4,23$ & 0,377 & 0,45 & $0,11-1,83$ & 0,264 \\
\hline \multicolumn{7}{|c|}{ Doença concomitante/Alergia * } \\
\hline Não & 1,00 & & & 1,00 & & \\
\hline Sim & 1,18 & $0,39-3,64$ & 0,768 & 1,31 & $0,24-7,11$ & 0,758 \\
\hline \multicolumn{7}{|l|}{ Eventos sistêmicos } \\
\hline \multicolumn{7}{|l|}{ Sexo } \\
\hline Masculino & 1,00 & & & 1,00 & & \\
\hline Feminino & 0,84 & $0,40-1,77$ & 0,650 & 3,29 & $1,25-8,68$ & 0,016 \\
\hline \multicolumn{7}{|l|}{ Idade (meses) } \\
\hline 6-11 & 1,00 & & & 1,00 & & \\
\hline $12-24$ & 0,62 & $0,29-1,32$ & 0,215 & 0,46 & $0,18-1,19$ & 0,108 \\
\hline \multicolumn{7}{|l|}{ Cor da pele } \\
\hline Branca & 1,00 & & & 1,00 & & \\
\hline Negra/Parda & 1,31 & $0,61-2,85$ & 0,489 & 0,53 & $0,21-1,36$ & 0,188 \\
\hline \multicolumn{7}{|c|}{ Doença concomitante/Alergia * } \\
\hline Não & 1,00 & & & 1,00 & & \\
\hline Sim & 2,76 & $1,11-6,89$ & 0,029 & 2,67 & $0,87-8,16$ & 0,085 \\
\hline
\end{tabular}

HR: hazard ratio; IC95\%: intervalo de 95\% de confiança.

* $80 \%$ doenças do sistema respiratório (sinusite, bronquite e asma), $20 \%$ se distribuem entre alergias dermatológicas, alimentares e medicamentosas. 
avaliaram a segurança deste imunobiológico em crianças de diferentes países.

No que se refere à maior frequência dos eventos adversos após a vacinação com a primeira dose, evidenciada neste trabalho, situação semelhante foi observada em estudos realizados no Canadá 22, Costa Rica 24 e nos Estados Unidos 25. Em uma investigação realizada na Holanda 23, a primeira vacinação mostrou-se associada à maior ocorrência, intensidade e duração da febre.

Com relação ao fato de os eventos sistêmicos terem sido mais comuns do que os locais, tanto na primeira quanto na segunda vacinação no presente trabalho, esse achado também foi identificado em outros estudos sobre a temática 13,22. Scheifele et al. ${ }^{22}$ acompanharam 167 crianças entre 6 e 35 meses após vacinação contra a influenza A (H1N1) 2009 e observaram maior frequência de eventos adversos sistêmicos comparados com os locais tanto na primeira $(76 \% v s .47 \%)$ quanto na segunda vacinação ( $61,8 \%$ vs. $36 \%)$, com associação estatisticamente significativa. Em um estudo realizado na Austrália 13 com 162 crianças, também, encontrou-se maior frequência de eventos adversos sistêmicos. Este fato pode estar relacionado à idade dos sujeitos do estudo, pois as manifestações sistêmicas são mais frequentes em indivíduos que não tiveram contato anterior com os antígenos da vacina, como, por exemplo, as crianças 11 .

Os eventos adversos locais mais frequentes nas crianças deste estudo após a vacinação foram dor e vermelhidão no local da injeção, e os sistêmicos foram irritabilidade, febre e vômito. Os eventos adversos mais frequentes observados em crianças pós-vacinação contra a influenza A (H1N1) 2009 não têm sido descritos de maneira uniforme na literatura científica. No estudo realizado por Scheifele et al. 22, foram encontrados dor e edema no local da injeção como reações locais e, irritabilidade e distúrbios do sono como reações sistêmicas mais comuns. Nolan et al. 13 identificaram como eventos mais frequentes dor no local da injeção, irritabilidade e febre em crianças entre 6 e 35 meses de idade. Mallory et al. 25 evidenciaram como eventos mais incidentes, em crianças pequenas, o vômito, a náusea e a diarreia. Portanto, há dificuldade em se obter uma vigilância padronizada de eventos adversos em diferentes estudos e áreas geográficas.

A febre foi um evento adverso pós-vacinação contra a influenza A (H1N1) 2009 com grande variação de frequência entre os estudos revisados. No presente trabalho, esse desfecho foi identificado como evento adverso pós-vacinação em $18,6 \%$ das crianças na primeira dose e em 16,1\% na segunda dose. No estudo realizado por Bross et al. 23 , a febre acometeu $56,2 \%$ dos vacinados após a primeira dose e $56,1 \%$ após a segunda, em crianças entre seis meses e quatro anos de idade. van't Klooster et al. 26 identificaram a febre como evento adverso em $29,6 \%$ das crianças após a primeira vacinação e em $30,7 \%$ após a segunda. No estudo de Scheifele et al. 22, a febre foi identificada em $3,6 \%$ das crianças após a primeira vacinação e em 8,6\% após a segunda. É interessante notar, no entanto, que a classificação da febre não estava clara em todos os trabalhos.

As associações entre crianças com doença concomitante/alergia, em especial doenças do sistema respiratório, com a ocorrência de algum tipo de evento adverso (HR = 3,43; IC95\%: 1,34$8,77)$ e eventos adversos sistêmicos $(\mathrm{HR}=2,76$; IC95\%: 1,11-6,89) foram estatisticamente significativas após a primeira dose da vacina. Assim, os riscos de apresentar algum tipo de evento adverso ou eventos adversos sistêmicos após a primeira dose da vacina foram, respectivamente, $243 \%$ e $176 \%$ maior em crianças com doença concomitante/alergia em comparação aquelas sem a enfermidade. Os eventos adversos foram de intensidade leve, na sua maioria, e com duração de até três dias, sendo essas características semelhantes nas crianças sem doença concomitante/alergia.

Estudos sobre a segurança das vacinas contra a influenza em pessoas com doenças respiratórias, principalmente a asma, não são raros, entretanto, são controversos. Os resultados apontam desde a segurança e indicação da vacina para essa população $27,28,29$ até dados sobre exacerbação das crises, aumento da tosse no período pós-vacinação e procura por serviços de saúde 30,31 .

Com relação à influenza A (H1N1) 2009, um estudo realizado para avaliar a segurança da vacina monovalente e sem adjuvante em indivíduos asmáticos e de diferentes idades não encontrou diferença na incidência de eventos adversos comparado com a população geral 29.

Nossos achados a respeito deste tópico devem ser interpretados com cautela, pois a mensuração da variável doença concomitante/alergia foi realizada a partir da informação dos pais ou responsáveis sobre o diagnóstico médico da enfermidade, além de não existir um grupo controle com indivíduos que não foram imunizados, por questões éticas. Por outro lado, esse resultado deve ser explorado em futuros estudos sobre a temática.

Cabe ressaltar, ainda, que a vacina apresentase como uma alternativa de prevenção, visto que crianças com diagnóstico de influenza pandêmica A (H1N1) 2009 tiveram a asma como fator de risco para a gravidade da doença e morte 32 . Assim, a vacinação, apesar dos eventos adversos, 
é vantajosa para essa população em comparação com as consequências da doença.

Em relação à intensidade dos eventos adversos manifestados pós-vacinação e avaliados neste estudo, observou-se a ocorrência de um evento adverso grave, possivelmente relacionado à vacinação, que foi febre $>39,5^{\circ} \mathrm{C}$ (com maior pico registrado de $39,8^{\circ} \mathrm{C}$ ) em criança com idade de vinte meses após imunização com a primeira dose da vacina. A febre alta foi relatada também como ocorrência de evento adverso grave pós-vacinação contra a influenza A (H1N1) 2009 por Nolan et al. 13 que identificaram o desfecho em uma das 82 crianças menores de três anos que foram imunizadas com a primeira dose da vacina. Em uma pesquisa realizada na Holanda, verificou-se que a intensidade da febre após a primeira dose da vacina foi associada com a decisão dos pais ou responsáveis em levar seus filhos para receber a segunda dose e, ainda, foi o evento que levou pais ou responsáveis a perder dia de trabalho 23 . A febre é um dos eventos adversos mais importantes, principalmente pela repercussão que pode ter na família da criança. Entretanto, pelo exposto, a ocorrência de eventos adversos graves pós-vacinação contra a influenza A (H1N1) 2009 é pouco frequente e os trabalhos concluíram que a segurança e reatogenicidade da vacina são aceitáveis e semelhantes aos eventos pós-vacinação contra a influenza sazonal administrada anualmente em diferentes países $6,12,13,14,20,21,22,23$.

Algumas considerações merecem destaque com relação às limitações deste estudo: (1) a generalização dos resultados deve ser vista com cautela, pois se trata de uma população de usuários de um serviço de saúde e não de uma amostra aleatória de base populacional; (2) o tamanho final da amostra não é suficiente para identificar a possibilidade de eventos raros que poderiam se tornar aparentes após administração da va- cina em massa; (3) o tamanho final da amostra, também, pode diminuir a representatividade da população estudada e o poder estatístico em virtude das perdas ocorridas, especialmente em relação à segunda dose da vacina. Todavia, essa é uma característica intrínseca à faixa etária das crianças do estudo, que é marcada por intensa vacinação com diversos imunobiológicos. A maior parte das perdas ocorreu para se manter a exposição somente à vacina contra influenza $\mathrm{A}$ (H1N1) 2009; (4) os resultados com relação aos eventos adversos após a segunda dose poderiam estar afetados pela exclusão de um maior número de crianças entre 6 e 11 meses de idade. Entretanto, essa variável não permaneceu associada ao desfecho após a análise multivariada dos dados; (5) as vacinas foram adquiridas de dois laboratórios diferentes, não sendo possível especificar a origem do imunobiológico administrado em cada sujeito. Esse fato também foi observado em outro estudo sobre a temática 12 ; (6) deve se considerar a possibilidade de viés de mensuração e classificação devido ao fato de os eventos adversos terem sido coletados a partir de observações dos pais ou responsáveis pelas crianças.

Por outro lado, destaca-se como potencialidade deste trabalho o seu desenho longitudinal que, dentre os estudos observacionais, é aquele que melhor garante a relação de causa e efeito entre as exposições e os desfechos investigados.

O presente estudo fornece elementos sobre os eventos adversos pós-vacinação contra influenza A (H1N1) 2009, permitindo o melhor conhecimento sobre a temática na população de crianças entre seis meses e dois anos de idade, apontando características, assim como possíveis fatores associados ao surgimento dos mesmos. Essas informações são relevantes para um processo de monitoramento desses eventos adversos, uma vez que constituem o principal instrumento para controle da segurança das vacinas. 


\section{Resumo}

O objetivo do estudo foi estimar a frequência e os fatores associados à ocorrência de eventos adversos pósvacinação contra a influenza pandêmica A (H1N1) 2009 em crianças com idade entre seis meses e dois anos. Participaram do estudo 156 crianças. Modelos multivariados de regressão de Cox foram construídos para avaliar a associação independente de cada covariável e a queixa de pelo menos um evento adverso. A força da associação foi medida pela hazard ratio e seus respectivos intervalos de 95\% de confiança. Após a primeira dose, foi relatado algum tipo de evento adverso por 40,3\% dos participantes e, após a segunda, por $35,5 \%$. Os eventos sistêmicos foram mais frequentes que os locais, destaque para irritabilidade, diarreia e febre. As incidências de eventos adversos, no geral e sistêmicos, após a primeira dose, foram maiores nas crianças com doença concomitante/alergia em relação àquelas sem o agravo (HR = 3,43; IC95\%: 1,34-8,77 e $H R=2,76$; IC95\%: 1,11-6,89). A maioria dos eventos foi de intensidade leve. Febre alta, vômito e diarreia motivaram a busca por serviços de saúde.

Vírus da Influenza A Subtipo H1N1; Vacinas; Efeitos Adversos; Criança

\section{Colaboradores}

G. N. Andrade contribuiu com a elaboração do projeto, coordenação do trabalho de campo, análise e interpretação dos dados, concepção e redação do artigo. A. M. Pimenta participou da análise e interpretação dos dados, concepção e redação do artigo. D. A. Silva participou da elaboração do projeto, trabalho de campo e concepção do artigo. A. M. F. Madeira contribuiu com a elaboração do projeto, coordenação do trabalho de campo e concepção do artigo.

\section{Agradecimentos}

Os autores são gratos a todos os funcionários do Centro de Saúde São Paulo, a Elffie de Andrade e Fabrícia Cecília Marques Ribeiro pela colaboração na fase de coleta de dados.

\section{Referências}

1. Secretaria de Vigilância em Saúde, Ministério da Saúde. Guia de vigilância epidemiológica, caderno 1 - influenza. Brasília: Ministério da Saúde; 2010.

2. Lanying D, Zhou Y, Jiang S. Research and development of universal influenza vaccines. Microbes Infect 2010; 12:280-6.

3. Programa Nacional de Imunizações, Departamento de Vigilância Epidemiológica, Secretaria de Vigilância em Saúde, Ministério da Saúde. Estratégia nacional de vacinação contra o vírus Influenza pandêmico (H1N1) 2009. Brasília: Ministério da Saúde; 2010.

4. Coordenadoria de Controle de Doenças, Secretaria de Estado da Saúde de São Paulo. Características dos casos notificados de Influenza A/H1N1. Rev Saúde Pública 2009; 43:900-4.

5. Secretaria de Vigilância em Saúde, Ministério da Saúde. Informe epidemiológico influenza pandêmica (H1N1) 2009. Brasília: Ministério da Saúde; 2009.
6. Zhu FC, Wang H, Fang HH, Yang JG, Lin XJ, Liang $\mathrm{XF}$, et al. A novel influenza A (H1N1) vaccine in various age groups. N Engl J Med 2009; 361:2414-23.

7. Joseph TFL, Yeung NCY, Choi KC, Cheng MYM, Tsui HY, Griffiths S. Factors in association with acceptability of A/H1N1 vaccination during the influenza A/H1N1 pandemic phase in the Hong Kong general population. Vaccine 2010; 28:4632-7.

8. Iskander J, Haber P, Herrera G. Monitoring vaccine safety during an influenza pandemic. Yale J Biol Med 2005; 78:261-71.

9. Ofri D. The emotional epidemiology of H1N1 influenza vaccination. N Engl J Med 2009; 361:2594-5.

10. Wong LP, Sam IC. Factors influencing the uptake of $2009 \mathrm{H} 1 \mathrm{~N} 1$ influenza vaccine in a multiethnic Asian population. Vaccine 2010; 28:4499-505.

11. Agência Nacional em Vigilância Sanitária. Estratégia de vacinação contra o vírus influenza pandêmica (H1N1) 2009 - Protocolo de vigilância de eventos adversos pós-vacinação. Brasília: Ministério da Saúde; 2010. 
12. Liang XF, Wang HQ, Wang JZ, Fang HH, Wu J, Zhu FC, et al. Safety and immunogenicity of 2009 pandemic influenza A H1N1 vaccines in China: a multicentre, double-blind, randomised, placebocontrolled trial. Lancet 2010; 375:56-66.

13. Nolan T, McVernon J, Skeljo M, Richmond P, Wadia $\mathrm{U}$, Lambert S, et al. Immunogenicity of a monovalent 2009 influenza A (H1N1) vaccine in infants and children: a randomized trial. JAMA 2010; 303:37-46.

14. Plennevaux E, Sheldon E, Blatter M, Reeves-Hoche MK, Denis M. Immune response after a single vaccination against 2009 influenza A H1N1 in USA: a preliminary report of two randomised controlled phase 2 trials. Lancet 2010; 375:41-8.

15. Bricks LF. Crianças saudáveis devem receber a vacina contra influenza? Pediatria (São Paulo) 2004; 26:49-58.

16. Lopes MH, Mascheretti M, Franco MM, Vasconcelos R, Gutierrez EB. Occurrence of early adverse events after vaccination against influenza at a Brazilian reference center. Clinics 2008; 63:21-6.

17. Departamento de Vigilância Epidemiológica, Secretaria de Vigilância em Saúde, Ministério da Saúde. Vigilância dos eventos adversos pós-vacinação: cartilha para trabalhadores de sala de vacinação. Brasília: Ministério da Saúde; 2003. (Série F. Comunicação e Educação em Saúde).

18. Departamento de Vigilância Epidemiológica, Secretaria de Vigilância em Saúde, Ministério da Saúde. Manual de vigilância epidemiológica de eventos adversos pós-vacinação. 2a Ed. Brasília: Ministério da Saúde; 2008.

19. World Health Organization. Vaccine safety and adverse events following immunization. http://www. who.int/immunization_monitoring/routine/ immunization_adverse/en (acessado em 02/Jun/ 2012).

20. Oh CE, Lee J, Kang JH, Hong YJ, Kim YK, Cheong $\mathrm{HJ}$, et al. Safety and immunogenicity of an inactivated split-virus influenza $\mathrm{A} / \mathrm{H} 1 \mathrm{~N} 1$ vaccine in healthy children from 6 months to $<18$ years of age: a prospective, open-label, multi-center trial. Vaccine 2010; 28:5857-63.

21. Manzoli L, De Vito C, Salanti G, D’Addario M, Villari P, Ioannidis JP. Meta-analysis of the immunogenicity and tolerability of pandemic influenza A 2009 (H1N1) vaccine. PLoS One 2011; 6:e24384.

22. Scheifele DW, Ward BJ, Dionne M, Vanderkooi O, Langley JM, Dobson S, et al. Evaluation of adjuvanted pandemic H1N12009 influenza vaccine after one and two doses in young children. Pediatr Infect Dis J 2011; 30:402-7.
23. Broos N, van Puijenbroek EP, van Grootheest K. Fever following immunization with influenza A (H1N1) vaccine in children: a survey-based study in the Netherlands. Drug Saf 2010; 33:1109-15.

24. Arguedas A, Soley C, Abdelnour A, Sales V, Lindert K, Della Cioppa G, et al. Assessment of the safety, tolerability and kinetics of the immune response to A/H1N1v vaccine formulations with and without adjuvant in healthy pediatric subjects from 3 through 17 years of age. Hum Vaccin 2011; 7:58-66.

25. Mallory RM, Malkin E, Ambrose CS, Bellamy T, Shi L, Yi T, et al. Safety and immunogenicity following administration of a live, attenuated monovalent $2009 \mathrm{H} 1 \mathrm{~N} 1$ influenza vaccine to children and adults in two randomized controlled trials. PLoS One 2010; 5:e13755.

26. van't Klooster TM, Kemmeren JM, Melker HE, Vermeer-de Bondt PE, van der Maas NA. Two doses of pandemic influenza A (H1N1) vaccine: tolerability in healthy young children in the Netherlands. Hum Vaccin 2011; 7:1048-54.

27. Castro M, Dozor A, Fish J, Irvin C, Scharf S, Scheipeter ME, et al. The safety of inactivated influenza vaccine in adults and children with asthma. $\mathrm{N}$ Engl J Med 2001; 345:1529-36.

28. Pedroza A, Huerta JG, Garcia ML, Rojas A, LópezMartínez I, Penagos M, et al. The safety and immunogenicity of influenza vaccine in children with asthma in Mexico. Int J Infect Dis 2009; 13:469-75.

29. Busse WW, Peters SP, Fenton MJ, Mitchell H, Bleecker ER, Castro M, et al. Vaccination of patients with mild and severe asthma with a 2009 pandemic H1N1 influenza virus vaccine. J Allergy Clin Immunol 2011; 127:130-7.

30. Izurieta HS, Haber P, Wise RP, Iskander J, Pratt D, Mink C, et al. Adverse events reported following live, cold adapted, intranasal influenza vaccine. JAMA 2005; 294:2720-5.

31. Bueving HJ, Bernsen RM, de Jongste JC, van Suijlekom-Smit LW, Rimmelzwaan GF, Osterhaus AD, et al. Does influenza vaccination exacerbate asthma in children? Vaccine 2004; 23:91-6.

32. O’Riordan S, Barton M, Yau Y, Read SE, Allen U, Tran D. Risk factors and outcomes among children admitted to hospital with pandemic H1N1 influenza. CMAJ 2010; 182:39-44.

Recebido em 09/Out/2011

Versão final reapresentada em 07/Abr/2012 Aprovado em 11/Jun/2012 